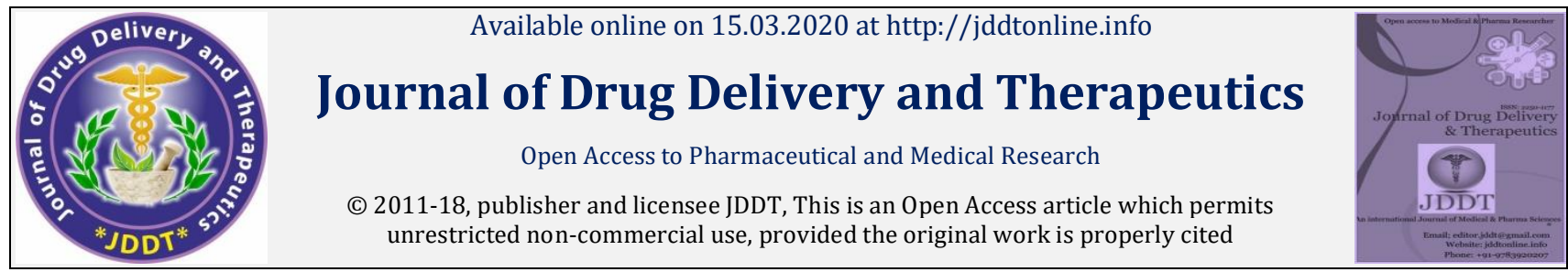

Open 2 Access

Research Article

\title{
Formulation and Evaluation of Pulsatile Drug Delivery System of Zafirlukast
}

\author{
A Vijaya Madhavi*, D Rama Brahma Reddy, M Venugopal, N Srihari, P Chenniah, P Koteswarao
}

Nalanda Institute of Pharmaceutical Sciences, Kantepudi (V), Sattenapalli (M), Guntur (D), AP, India

\begin{abstract}
In the current scenario of pharmaceutical research much attention has been focused on patients health in terms of therapeutic efficacy and economical standards (price factor).The formulation design consist of core tablets designed by direct compression method. Core tablets were coated with a naturally occurring swelling agent (carbopol \& Karaya gum). Evaluation studies were performed for prepared pulsatile tablets hardness. In in vitro release profile of 6 hours study in first 5 hours it shows minimum drug release and at the end of six hours rapid and transient release was observed. Stability studies proved that coating of tablets seems to decrease the effect of temperature and moisture on degradation of Zafirlukast. The pulsatile release has been achieved from tablet over a 7-8 hour period.
\end{abstract}

Objectives:

1. To Prepare and evaluate the pulsatile drug delivery system of zafirlukast.

2. To perform in-vitro drug release studies and to assess drug release studies.

Methodology: Methodology of the present research include the preparation of core tablet by Direct compression method. Preparation of coated tablet by preparing $16 \mathrm{~mm}$ die, placing the core tablet manually at center and compression was done in rotary compression tablet machine using $16.4 \times 8 \mathrm{~mm}$ flat oval shape punch.

Results: Pre-compression parameters were conducted for all formulations blend and were found to be satisfactory. Bulk density was found in the range $0.340-0.384 \mathrm{~g} / \mathrm{sq} \mathrm{cm}$ and tapped density in the range of $0.394-0.434 \mathrm{~g} / \mathrm{sq} \mathrm{cm}$. Using these two density factors Hausner's ratio and compressibility index was calculated. The powder blend of all formulations had Hausner's ratio was between 1.11 - 1.17 which indicates better flow property and compressibility index between 10.74 to 14.88 which indicates fair flow ability property. The fair flow ability property of the powder blend was also evidenced with angle of repose between $25.24-27.97$ which is below 40 indicating good flow ability.

Conclusion: A satisfactory attempt was made towards the development of efficacious drug delivery systems with already existing active ingredient (Zafirlukast). The coating given by the combination of carbopol and Karayagum enhanced the drug release from the coated tablet and side effects were minimized.

Keywords: Zafirlukast, Swelling Agent, Swelling index, Lag time, karaya gum.

Article Info: Received 04 Jan 2020; Review Completed 19 Feb 2020; Accepted 26 Feb 2020; Available online 15 March 2020

\section{Cite this article as:}

Madhavi AV, Brahma Reddy DR, Venugopal M, Srihari N, Chenniah P, Koteswarao P, Formulation and Evaluation of Pulsatile Drug Delivery System of Zafirlukast, Journal of Drug Delivery and Therapeutics. 2020;10(2):122-128 http://dx.doi.org/10.22270/jddt.v10i2.3983

*Address for Correspondence:

Dr. A. Vijaya Madhavi, Pharm.D, Assistant Professor, Dept. of Pharmacy Practice, Nalanda Institute of Pharmaceutical Scienc es, Kantepudi (V), Sattenapalli (M), Guntur (D), Andhra Pradesh, India.

\section{INTRODUCTION:}

In the current scenario of pharmaceutical research much attention has been focused on patient's health in terms of therapeutic efficacy and economical standards (price factor).The major goal for the drug delivery research is turned towards the development of efficacious drug delivery systems with already existing active ingredients.

Pulsatile drug delivery system: Pulsatile drug delivery system is most interesting and time specific system as per pathophysiological need of the disease. Pulsatile drug delivery system is characterized by time period of no release (lag time) followed by a rapid and complete drug release. The drug release was influenced by type of pulsatile delivery mechanism employed in the formulation (Capsular system, Osmotic system, rupturable polymeric coating). The lag time was reduced by replacing the swelling agents and disintegrating agents. By reducing the lag time the drug release was done before the actual time of release 1 .

Swelling agent ${ }^{2}$ : Swelling agent is used in the designing of dosage form to reduce the frequency of dosing by modifying the rate of drug release kinetics as well as dry absorption. It 
was proven that naturally occurring polymers are more efficient compared to chemical and synthetic in terms of patient health. The natural swelling agent employed in the formulation is Karaya gum.

The swelling behavior of karaya gum depends upon the presence of acetyl groups in its structure. Karaya gum swells in $60 \%$ alcohol, but remains insoluble in other organic solvents karaya may absorb up to 100 times its weight in water ${ }^{5}$. The major use of karaya gum is as a bulk laxative in view of its ability to form a mucilaginous gel on contact with water.

Swelling index: The extent of swelling was measured in terms of $\%$ weight gain by the granules. The swelling behavior of all formulations was studied. 2 gram from each formulation was kept in a Petri dish containing $\mathrm{pH} 6.8$ phosphate buffers. At the end of 1 hour, the Petri dish along with the granules was weighed. The weight of the granules, were noted, and the process was continued till the end of 24 hours. The swollen granules were weighed $\left(\mathrm{W}^{2}\right)$ and the percentage of swelling was calculated by the following equation 6 .

\section{Swelling index $=\left(\mathrm{W}^{2}-\mathrm{W}^{1}\right) \backslash \mathrm{W}^{1 *} \mathbf{1 0 0}$}

$\mathrm{W}^{2}=$ weight after absorption

$\mathrm{W}^{1}=$ weight before absorption

\section{MATERIALS AND METHODS:}

Materials: Zafirlukast, Polyplasdone XL 10, Ac-Di-Sol, Primojel, Micro crystalline cellulose, Talc, Magnesium stearate, Carbopol, Karaya gum. Other excipients are procured from Narmada chemicals.

\section{Formulation of Compressed Tablets of Zafirlukast}

The methodology adopted includes:

- Preparation of core tablet of Zafirlukast.

- Coating of the core tablets

1. Formulation of core tablet of Zafirlukast: The inner core tablets were prepared by using direct compression method as per the developed formulation table Accurately weighed amounts of Zafirlukast, MCC, Polyplasdone XL 10, Ac-Di-Sol, Primojel, and Talc were dry blended for about $15 \mathrm{~min}$ followed by addition of magnesium stearate. The mixture was then further blended for $10 \mathrm{~min}$. Now the resultant powder blend was manually compressed using punching machine and finally the core tablet was obtained.

2. Formulation of coated tablets of Zafirlukast: The optimized core tablets were coated with coating ingredients like Carbopol, Karaya gum. Now accurately weighed amount of barrier layer material was transferred into a $16 \mathrm{~mm}$ die then the core tablet was placed manually at the center. The remaining amount of the barrier layer material was added into the die and compressed. Compression of tablets was done in rotary compression tablet machine using $16.4 \times 8 \mathrm{~mm}$ flat oval shape punch. The prepared tablet of each batch was evaluated for the tablet properties.

\section{Evaluation of Formulations:}

1. Compatibility Studies: Compatibility with excipients was confirmed by FTIR studies. The pure drug and polymers were subjected to FTIR studies. In the present study, the potassium bromide disc (pellet) method was employed.
2. Preparation of Standard Calibration Curve of Zafirlukast in 0.1 N HCL: 100mg of zafirlukast was accurately weighed and transferred into $100 \mathrm{ml}$ volumetric flask. It was dissolved and diluted to volume with $0.1 \mathrm{~N}$ HCL buffer to give stock solution containing $1000 \mu \mathrm{g} / \mathrm{ml}$. The standard stock solution was then serially diluted with $0.1 \mathrm{~N} \mathrm{HCL}$ buffer to get 2 to $10 \mu \mathrm{g} / \mathrm{ml}$ of zafirlukast. The absorbance of the solution was measured against $0.1 \mathrm{~N} \mathrm{HCL}$ buffer as blank at $230 \mathrm{~nm}$ using UV visible spectrophotometer. The absorbance values were plotted against concentration $(\mu \mathrm{g} / \mathrm{ml})$ to obtain the standard calibration curve.

\section{Evaluation of Pre-Formulation Parameters:}

1. Angle of repose: The angle of repose of powder blend was determined by the funnel method. The accurately weight powder blend were taken in the funnel. The height of the funnel was adjusted in such away the tip of the funnel just touched the apex of the powder blend. The powder blend was allowed to flow through the funnel freely onto the surface. The diameter of the powder cone was measured and angle of repose was calculated by using fallowing equation.

$$
\operatorname{Tan} \theta=\mathbf{h} / \mathbf{r}
$$

Where, $\mathrm{h}$ and $\mathrm{r}$ are the height and radius of the powder cone respectively.

2. Determination of Bulk Density and Tapped Density: $20 \mathrm{~g}$ of the granules (W) from each formula were introduced into a $100 \mathrm{mlmeasuring}$ cylinder, and the initial volume was observed. The cylinder was allowed to fall under its own weight onto a hard surface from the height of $2.5 \mathrm{~cm}$ at $2 \mathrm{Sec}$ intervals. The tapping was continued until no further change in volume was noted. The bulk density, and tapped density were calculated using the following formulae.

\section{Bulk density $=\mathrm{W} / \mathrm{V}_{0}$}

\section{Tapped density $=\mathrm{W} / \mathrm{V}_{\mathbf{F}}$}

Where, $\mathrm{W}=$ weight of the granules,

$\mathrm{V}_{0}=$ initial volume of the granules,

$\mathrm{V}_{\mathrm{F}}=$ Zafirlukast volume of the granules

3. Hausner's Ratio

4. Compressibility index (Carr's Index)

Evaluation of tablet properties:

1. Weight variation: The weight of the tablet being made was routinely determined to ensure that tablet contains the proper amount of drug. The USP weight variation test is done by weighing 20tablets individually, calculating the average weight and comparing the individual weights to the average. The tablets met the USP specification that not more than 2 tablets are outside the percentage limits and no tablet differs by more than 2 times the percentage limit.

2. Tablet hardness: The resistance of tablets to shipping or breakage under conditions of storage, transportation and handling before usage depends on its hardness. The hardness of each batch of tablet was checked by using Monsanto hardness tester. The hardness was measured in terms of $\mathrm{kg} / \mathrm{cm}^{2} .3$ tablets were chosen randomly and tested for hardness. The average hardness of 3 determinations was recorded.

3. Friability: 20 tablets were weighed and the initial 
weight of these tablets was recorded and placed in Roche friabilator and rotated at the speed of $25 \mathrm{rpm}$ for 100 revolutions. Then tablets were removed from the friabilator, dusted off the fines and again weighed and the weight was recorded.

4. Tablet thickness: Thickness of the tablet is important for uniformity of tablet size. Thickness was measured using vernier Callipers. It was determined by checking the thickness often tablets of each formulation.

5. Content Uniformity: The tablets were tested for their drug content uniformity At random 20tablets were weighed and powdered. The powder equivalent to $200 \mathrm{mg}$ was weighed accurately and dissolved in $100 \mathrm{ml}$ of buffer used. The solution was shaken thoroughly. The un dissolved matter was removed by filtration through Whattman's filter paper No.41. Then the serial dilutions were carried out. The absorbance of the diluted solutions was measured at $230 \mathrm{~nm}$. The concentration of the drug was computed from the standard curve of the Zafirlukast in 6.8 phosphate buffer.

6. Disintegration time: Tablet disintegration is an important step in drug absorption. The test for disintegration was carried out in Electro lab USP disintegration test apparatus. It consists of 6glasstubes which are 3inches long, open at the top, and held against a1 0mesh screen, at the bottom end of the basket rack assembly. To test the disintegration time of tablets, one tablet was placed in each tube and the basket rack was position Edina 1 litre beaker containing 6.8 phosphate buffer solution at $37^{\circ} \mathrm{C} \pm 1^{\circ} \mathrm{C}$ such that the tablet remains $2.5 \mathrm{~cm}$ below the surface of the liquid. The time taken for the completed is integration of the tablets was noted.

7. In vitro Dissolution time: In-vitro dissolution study of core and coated tablets of Zafirlukast was carried out using Electro lab TDT-08L USP dissolution test apparatus. The details are given as below:

Procedure: Tablet was introduced into the basket of the Electrolab TDT-08L USP dissolution test apparatus and the apparatus was set in motion, $5 \mathrm{ml}$ of sample was withdrawn for half a $\mathrm{n}$ hour at $5 \mathrm{~min}$ intervals. Samples withdrawn were analyzed by UV-spectrophotometer for presence of drug using buffer solution as blank.

\section{Evaluation of Pulsatile Drug Delivery Systems:}

1. Characteristics of coated tablets of Zafirlukast: Characteristics of tablets of Zafirlukast such as hardness and disintegration test were conducted. 3 tablets were taken and hardness off or formulations was determined by using Monsanto hardness tester. Average of three determinations was noted down. 6 tablets were taken in Electrolab USP Disintegration test apparatus and disintegration time of tablets was determined using pH 6.8 buffer. Thickness of coated Zafirlukast tablet formulations was determined by using digital Vernier calipers. 3 tablets of each type of coated formulation were determined for thickness and average thickness of the formulation was determined. Similarly the thickness of the coating on the formulation was determined by deducting the thickness of core tablets from thickness of the coated formulation. A successful Pulsatile drug delivery system is one that remains intact in the physiological environment of stomach and small intestine for upto six hours, releasing no or minimum amount of drug, but completely releases the drug after six hours.
2. In-vitro Dissolution methods: Dissolution testing of pulsatile delivery systems with the conventional paddle method at $50 \mathrm{rpm}$ and $37 \pm 0.5^{\circ} \mathrm{Ch}$ as usually been conducted in different buffers for different periods of time to simulate the GI tract $\mathrm{pH}$ and transit time that the pulsatile delivery system might encounter in-vivo .The ability of the coats/carrier store main intact in the physiological environment of the stomach and small intestine is generally assessed by conducting drug release studies in $0.1 \mathrm{~N}$ HCL for 2 hours (mean gastric emptying time) and in pH6. 8phosphate buffer for remaining hours (mean small intestinal transit time) using USP dissolution rate test apparatus. The samples were withdrawn at regular intervals and analyzed by UV spectrophometer (Shimadzu UV/Vis 1800 ) for the presence of the drug. Dissolution tests were performed in triplicate. Despite the simplicity and convenience, convention al dissolution testing primarily provides essential information on the processing specifications of a pulsatile drug delivery system rather than on the validity of the system design.

3. Stability Studies: In the present study optimized formulation was selected for the study and formulations were packed in amber-colored bottles tightly plugged with cotton and capped.

They were exposed to $40^{\circ} \mathrm{C}$ temp and $75 \% \mathrm{RH}$ for 30days.At regular intervals, the tablets were taken in $100 \mathrm{ml}$ of $\mathrm{pH}$ 6.8 buffer and were shaken for $1 \mathrm{hr}$.The resultant solutions were filtered, properly diluted and estimated spectro photometrically by keeping pH6.8buffer as blank .\% drug remained under composed was checked for both core and coated tablets.

\section{RESULTS \& DISCUSSION:}

Pre compression parameters of core tablet of zafirlukast: Pre-compression parameters were conducted for all formulations blend and were found to be satisfactory. Bulk density was found in the range $0.340-0.384 \mathrm{~g} / \mathrm{sqcm}$ and tapped density in the range of $0.394-0.434 \mathrm{~g} / \mathrm{sqcm}$. Using these two density factors Hausner's ratio and compressibility index was calculated. The powder blend of all formulations had Hausner's ratio was between $1.11-1.17$ which indicates better flow property and compressibility index between 10.74 to 14.88 which indicates fair flow ability property. The fair flow ability property of the powder blend was also evidenced with angle of repose between $25.24-27.97$, which is below 40 indicating the good flow ability.

\section{Pre compression parameters of core tablets:}

1. Weight Variation Test: The percentage weight variations for all formulations were given. All the formulated (F1 to F9) tablets passed weight variation test as the $\%$ weight variation was within the pharmacopoeia limits. The weights of all the tablets were found to be uniform with low standard deviation values.

2. Hardness test: The measured hardness of tablets of all the formulations ranged between $3-4 \mathrm{~kg} / \mathrm{cm} 2$. This ensures good handling characteristics of all batches.

3. Disintegration test for core tablets: It was found between 30 - 86 seconds ensuring that all the cores of different formulations were rapid disintegrating type.

4. Friability Test: The $\%$ friability was less than $0.77 \%$ in all the formulations ensuring that the tablets were 
mechanically stable.

Evaluation of Physical parameters of compressed tablets of Zafirlukast

1. Weight Variation Test: The percentage weight variations for all formulations were given. All the formulated (Z1F9 to Z6F9) tablets passed weight variation test as the $\%$ weight variation was within the pharmacopoeia limits. The weights of all the tablets were found to be uniform with low standard deviation values.

2. Hardness test: The measured hardness of tablets of all the formulations ranged between $5.12-5.30$ $\mathrm{kg} / \mathrm{cm} 2$. This ensures good handling characteristics of all batches.

3. Thickness: The measured thickness of tablets of all the formulations ranged between $4.65-4.85 \mathrm{~mm}$. This ensures good handling characteristics of all batches.

4. Friability Test: The $\%$ friability was less than $1 \%$ in all the formulations ensuring that the tablets were mechanically stable.

5. Content uniformity: The percentage of drug content for F1 to F9 was found to be between $90.23 \%$ $98.86 \%$ it complies with official specifications. The percentage of drug content for $\mathrm{Z1F9}$ to $\mathrm{Z} 6 \mathrm{~F} 9$ was found to be between $93.30 \%$ - 99.71\%. It complies with official specifications.

6. Stability studies: Stability Studies were carried out at $40^{\circ} \mathrm{C}$ tempand75\%RH for 30 days. The core tablet and coated tablet of selected formulation were packed in amber-colored bottles tightly plugged with cotton and capped. And \% drug content was checked at regular time intervals.
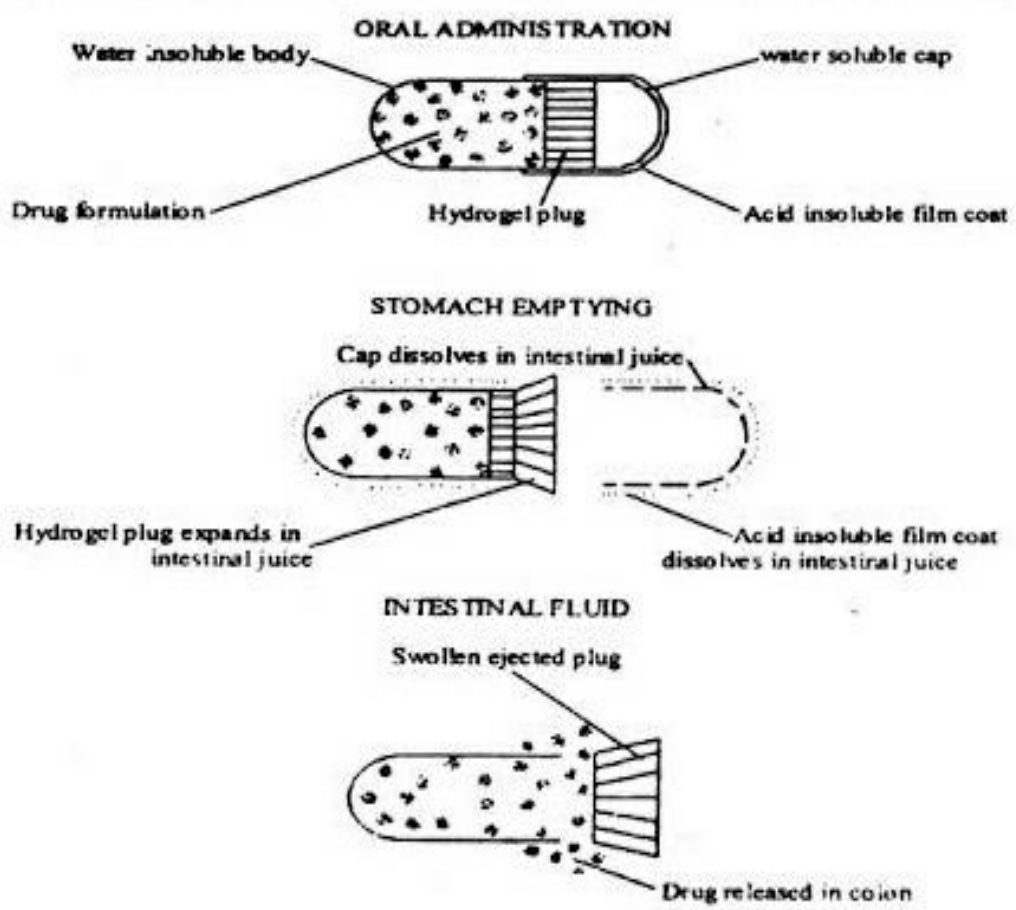

Fig .1 Releasemechanisam of pulsatile drug delivery system

Table 1: Formulation table of core tablets:

\begin{tabular}{|c|c|c|c|c|c|c|c|c|c|}
\hline Ingredients & F1 & F2 & F3 & F4 & F5 & F6 & F7 & F8 & F9 \\
\hline Zafirlukast & 10 & 10 & 10 & 10 & 10 & 10 & 10 & 10 & 10 \\
\hline Ac-Di-Sol & 5 & 7.5 & 12.5 & -- & -- & -- & -- & -- & -- \\
\hline Primojel & -- & -- & -- & 5 & 7.5 & 12.5 & -- & -- & -- \\
\hline Polyplasdone XL 10 & -- & -- & -- & -- & -- & -- & 5 & 7.5 & 12.5 \\
\hline Micro crystalline cellulose & Q.S & Q.S & Q.S & Q.S & Q.S & Q.S & Q.S & Q.S & Q.S \\
\hline Magnesium.stearate & 4 & 4 & 4 & 4 & 4 & 4 & 4 & 4 & 4 \\
\hline Talc & 2 & 2 & 2 & 2 & 2 & 2 & 2 & 2 & 2 \\
& & & & & & & & & \\
\hline
\end{tabular}


Table 2: Composition of compressed coated tablets:

\begin{tabular}{|c|c|c|c|c|c|c|}
\hline Formulation & Z1F9 & Z2F9 & Z3F9 & Z4F9 & Z5F9 & Z6F9 \\
\hline Core & 100 & 100 & 100 & 100 & 100 & 100 \\
\hline Carbopol & 400 & & 200 & 150 & 250 & 275 \\
\hline Karaya gum & & 400 & 200 & 250 & 150 & 125 \\
\hline Total weight & 500 & 500 & 500 & 500 & 500 & 500 \\
\hline
\end{tabular}

Table: 3 In - Vitiro release profile of Formulation (F1-F9):

\begin{tabular}{|c|c|c|c|c|c|c|c|c|c|}
\hline TIME & F1 & F2 & F3 & F4 & F5 & F6 & F7 & F8 & F9 \\
\hline 0 & 0 & 0 & 0 & 0 & 0 & 0 & 0 & 0 & 0 \\
\hline 5 & 16.78 & 19.96 & 24.47 & 22.59 & 28.88 & 34.40 & 40.11 & 43.26 & 47.13 \\
\hline 10 & 25.57 & 27.78 & 33.56 & 30.48 & 36.60 & 42.26 & 52.74 & 59.69 & 62.22 \\
\hline 15 & 34.49 & 39.90 & 42.02 & 41.79 & 49.98 & 53.36 & 63.60 & 66.78 & 70.65 \\
\hline 20 & 52.11 & 57.15 & 61.19 & 50.80 & 65.54 & 72.20 & 75.09 & 79.04 & 84.14 \\
\hline 25 & 69.90 & 73.30 & 76.62 & 61.60 & 79.59 & 84.47 & 82.97 & 87.23 & 91.10 \\
\hline 30 & 76.65 & 82.20 & 85.59 & 72.79 & 88.80 & 96.66 & 87.58 & 92.41 & 99.45 \\
\hline 45 & 87.19 & 91.16 & 97.40 & 84.63 & 97.19 & & 93.30 & 98.91 & \\
\hline 60 & 95.50 & 98.07 & & 92.77 & & & 99.78 & & \\
\hline
\end{tabular}

Table 4: Cumulative \%drug release of coated different formulation (Z1F9 to Z6F9):

\begin{tabular}{|c|c|c|c|c|c|c|}
\hline Time(HRS) & Z1F9 & Z2F9 & Z3F9 & Z4F9 & Z5F9 & Z6F9 \\
\hline 0 & 0 & 0 & 0 & 0 & 0 & 0 \\
\hline 1 & 0.54 & 0.75 & 0.41 & 0.55 & 0.89 & 0.22 \\
\hline 2 & 0.63 & 0.98 & 0.77 & 1.23 & 1.36 & 0.64 \\
\hline 3 & 2.03 & 39.78 & 3.69 & 5.60 & 4.47 & 0.84 \\
\hline 4 & 4.12 & 79.96 & 8.79 & 10.23 & 9.98 & 1.97 \\
\hline 5 & 19.65 & 95.64 & 26.65 & 21.36 & 26.6 & 13.36 \\
\hline 6 & 32.30 & -- & 48.87 & 34.45 & 47.48 & 74.46 \\
\hline 7 & 44.47 & -- & 66.30 & 66.54 & 69.14 & 85.97 \\
\hline 8 & 74.12 & -- & 97.90 & 80.21 & 87.19 & 98.89 \\
\hline 9 & 84.20 & -- & -- & 96.54 & 100.01 & -- \\
\hline 10 & 95.46 & -- & -- & -- & -- & -- \\
\hline
\end{tabular}


Table 5: in-vitro drug release mechanism of best core formulation:

\begin{tabular}{|c|c|c|}
\hline Batch & Zero order & First order \\
\hline Code & $\mathrm{r}^{2}$ & $\mathrm{r}^{2}$ \\
\hline F9 & 0.872 & 0.982 \\
\hline
\end{tabular}

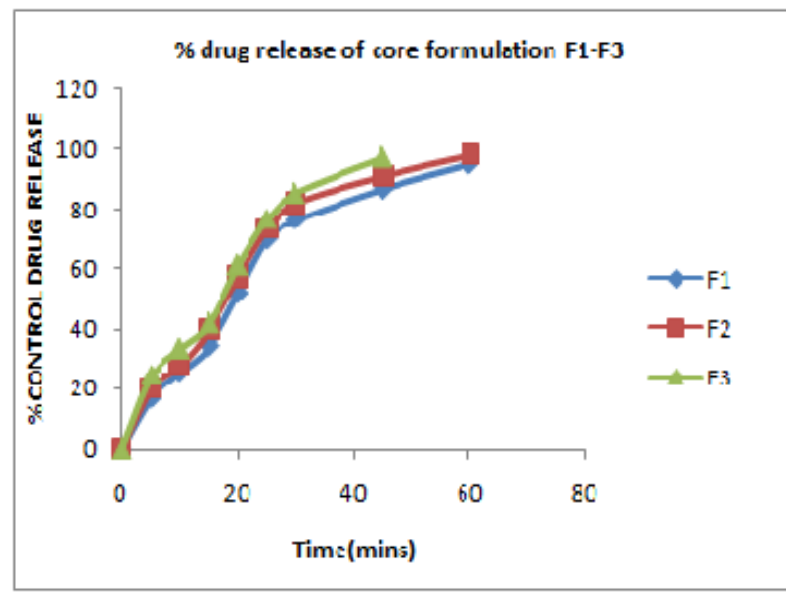

Fig-2 Cumulative percentage of formulation F1-F3

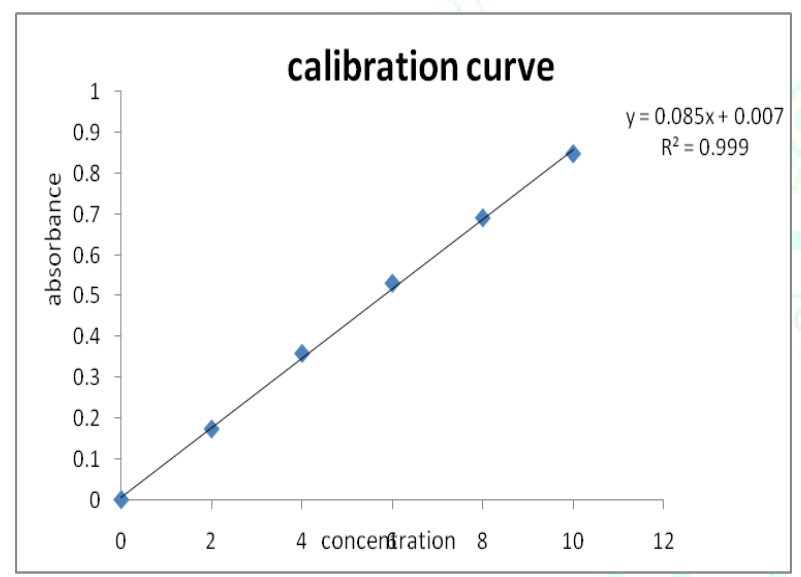

Fig-3 Calibration curve of formulation F1-F3

Drug release of core formulation F1-F3

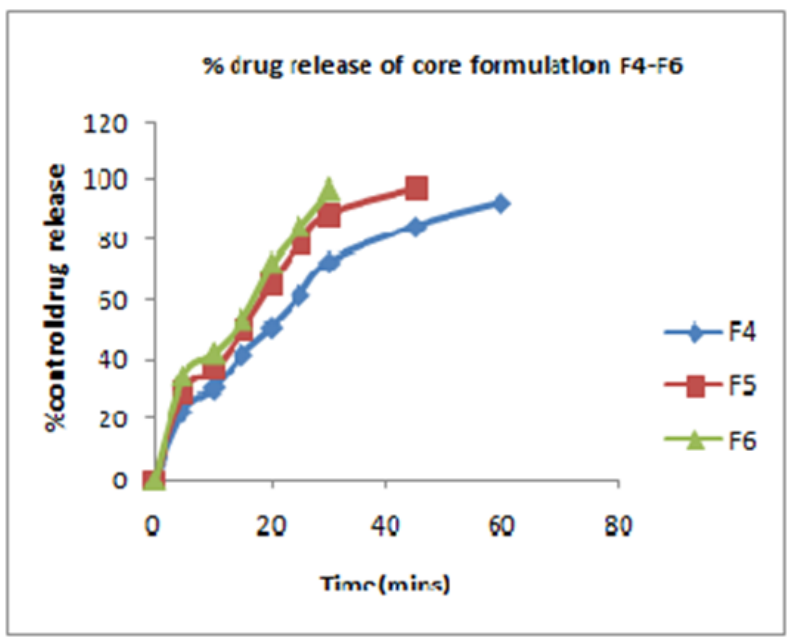

Fig-4 Cumulative percentage drug Release of core formulation F4-F6

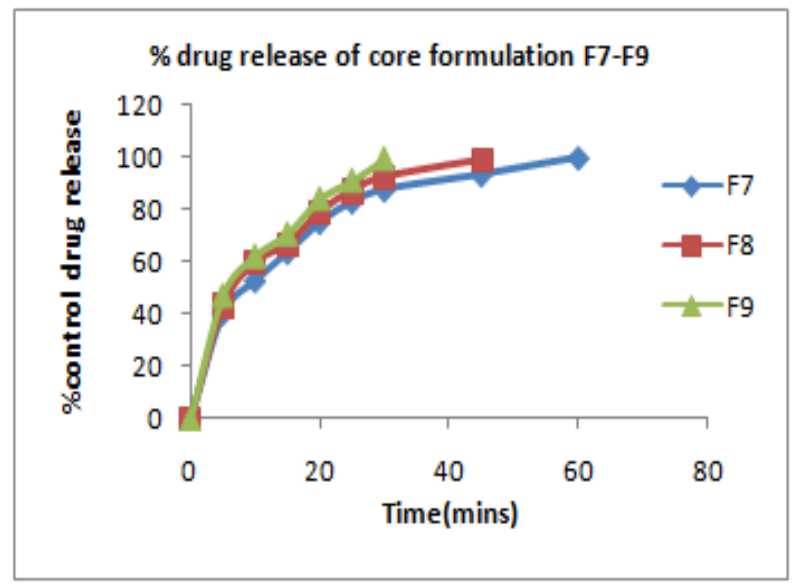

Fig-5 Cumulative Percentage drug release of core formulation F7-F9

Drug release kinetics mechanisms of best formulation (F9):

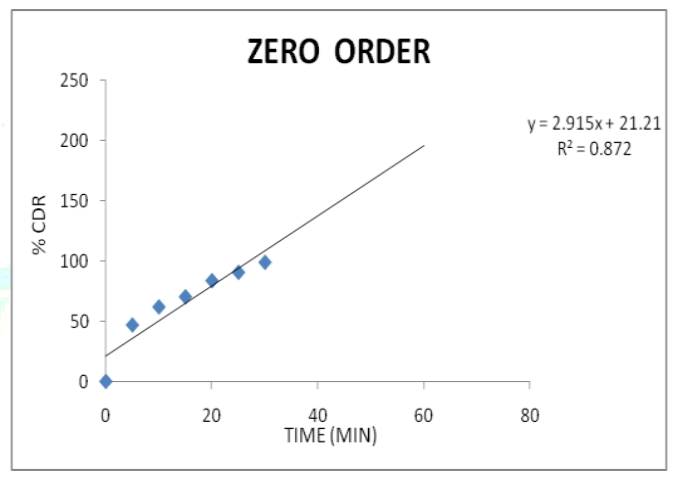

Fig-6: Zero order release kinetics for F9

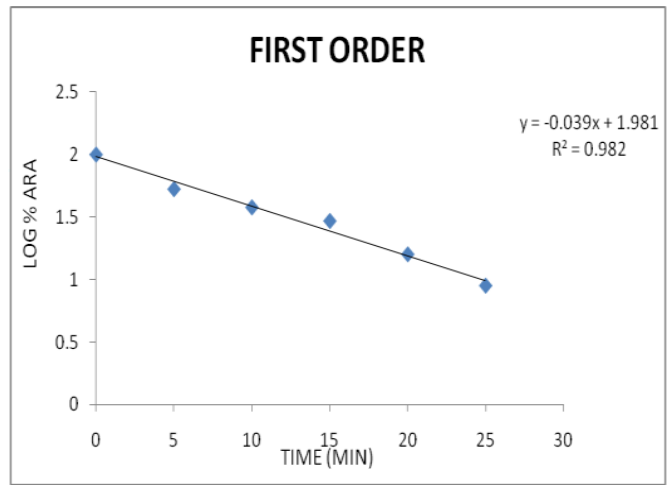

Fig-7: First order release kinetics for F9

Table 6: Showing \% Drug Content during storage

\begin{tabular}{|c|c|c|}
\hline $\begin{array}{c}\text { Time in } \\
\text { Days }\end{array}$ & $\begin{array}{c}\text { \%Drug Content in } \\
\text { Core Tablets }\end{array}$ & $\begin{array}{c}\text { \%Drug Content in } \\
\text { Coated Tablets }\end{array}$ \\
\hline 0 & 98.86 & 99.71 \\
\hline 10 & 98.79 & 99.75 \\
\hline 20 & 98.81 & 99.70 \\
\hline 30 & 99.84 & 99.69 \\
\hline
\end{tabular}




\section{CONCLUSION:}

A satisfactory attempt was made towards the development of efficacious drug delivery systems with already existing active ingredient (Zafirlukast). The coating given by the combination of carbopol and Karayagum enhanced the drug release from the coated tablet and side effects were minimized. On the basis of drug content, in-vitro release studies and its kinetic data F9 of core tablet and Z5F9 of were selected as optimized formulations for designing pulsatile device. Finally from the above results we can conclude that pulsatile drug delivery system of Zafirlukast can be formulated using above mentioned polymers.

\section{REFERENCES:}

1. Jaiswal H, Ansari VA, Pandit JN, Ahsan F, pulsatile drug delivery system: An Overview with special Emphasis on Losartan and Captopril, Research Journal of Pharmacy and Technology. 2019; 12(7):3175. 10.5958/0974360X.2019.00535.3.

2. Kilkuchi A and Okano T; "Pulsatile drug release control using hydrogels "; Advan. Drug Del. Reviews, 2002; 54:53-77.

3. Maurya SD, Arya RKK, Rajpal G, Dhakar RC, Self-micro emulsifying drug delivery systems (SMEDDS): a review on physico-chemical and biopharmaceutical aspects, Journal of Drug Delivery and Therapeutics 2017; 7(3):55-65

4. Okano T, Iskakov RM, Kikuchi A, Time-programmed pulsatile release of dextran from calciumalginate gel beads coated with carboxy-n-propylacrylamide copolymers. J. Controlled Release, 2002; 80:57-68.

5. Martin A, Physical Pharmacy-physiochemical principles in the pharmaceutical sciences. 4th Ed. New Delhi: B.I Waverly Pvt. Ltd; 1996; 313-31.

6. Liberman H, Lachman L. The Theory and Practice of Industrial Pharmacy. IIIrd ed.Bombay: Verghese Publication House; 1991; 171-193.

7. Momin A, Khan S, Ghadage DM, Yadav AV, Wagh A, Formulation and evaluation of bilayer tablets of propranolol hydrochloride, Journal of Drug Delivery and Therapeutics 2017; 7(2):50-57

8. Shivakumar HG, Pramod KTM, Kashappa GD. Pulsatile drug delivery systems.ssIndian J Pharm Educ. 2003 July-Sept; 37(3):125-128.

9. Robert W, Thomas P. Drug therapy for hypercholestemia and Dyslipidemia.In: Goodmen and Gilmans. The pharmacological basis of therapeutics.10th ed. 2003: McGraw- hill. Medical publishing division p. 971-972.

10. Patel KB, Vyas JR, Upadhyay UM, Formulation and evaluation of sustained release matrix tablets of nateglinide, ournal of Drug Delivery and Therapeutics 2015; 5(5):19-25

11. Gothaskar AV, Joshi AM, Joshi NH. Pulsatile drug delivery system-A review.DrugDel Tech. 2004 June; 4(5).

12. Anal AK. Time-Controlled Pulsatile Delivery Systems for Bioactive Compounds.Recent Patents on Drug Delivery \& Formulation.2006 Dec; 1:73-79.vitro/in vivo evaluationReview. I J Pharm. 2002; 253:1-15. 